\title{
Do Short-Range Correlations Cause the Nuclear EMC Effect in the Deuteron?
}

\author{
X. G. Wang $\odot,{ }^{1}$ A. W. Thomas $\odot,{ }^{1}$ and W. Melnitchouk $\oplus^{2}$ \\ ${ }^{1}$ CSSM and ARC Centre of Excellence for Particle Physics at the Terascale, Department of Physics, \\ University of Adelaide, Adelaide, South Australia 5005, Australia \\ ${ }^{2}$ Jefferson Lab, Newport News, Virginia 23606, USA
}

(Received 14 April 2020; revised 9 November 2020; accepted 4 December 2020; published 31 December 2020)

\begin{abstract}
The relative contributions to the valence nuclear European Muon Collaboration (EMC) effect in the deuteron arising from nucleon off-shell effects and Fermi motion are examined in models which include nuclear binding and off-shell effects. Contrary to expectations, the effect of Fermi motion overwhelms the off-shell effects for nucleons in short-range correlations (SRCs), calling into question the hypothesized causal connection between SRCs and the EMC effect.
\end{abstract}

DOI: 10.1103/PhysRevLett.125.262002

The discovery by the European Muon Collaboration (EMC) of the unexpected suppression of the deep-inelastic structure functions of atomic nuclei in the valence quark region [1-4], which we shall refer to as the "valence EMC effect," still has no widely accepted explanation after more than three decades [5-8]. Experimental advances have yielded precise data across the periodic table $[9,10]$, while many theoretical ideas have been proposed, ranging from nucleon "swelling" [11,12] to enhancement of the pion field [13-15], off-shell effects [16], suppression of pointlike configurations [17], and modification of nucleon structure in the strong scalar and vector mean fields occurring in nuclei [18-22]. In a few cases, new predictions have been made against which some of these ideas may be tested in future experiments [23,24], but to date a universally accepted explanation has remained stubbornly elusive.

In the past few years, there has been remarkable progress at Jefferson Lab in clearly identifying the tensor force as the prime source of short-range correlations (SRCs) in nuclei [25]. Following that success, the observation of a correlation between the size of the valence EMC effect and the number of nucleons in SRCs led to suggestions that SRCs may be the underlying source of the effect [26]. The physical motivation is that, in deep-inelastic scattering (DIS) involving nucleons in SRCs, the spectator to the DIS event will have a relatively high kinetic energy, which forces the struck nucleon to be far off-mass-shell. Within this picture, it was shown that one could extract an approximately "universal function," applicable to all nuclei

Published by the American Physical Society under the terms of the Creative Commons Attribution 4.0 International license. Further distribution of this work must maintain attribution to the author(s) and the published article's title, journal citation, and DOI. Funded by SCOAP. including the deuteron, which describes the modification of the structure function of such a far off-shell nucleon.

In this Letter, we examine the proposal that SRCs may be responsible for the valence EMC effect in more detail. In order to place the theoretical discussion on a quantitative level, we use a general class of phenomenologically successful models of DIS from nuclei, which take into account nuclear binding, Fermi motion, and nucleon off-shell effects. The key feature of these models is that, irrespective of their details, they allow these effects to be separated into those arising from low- and highmomentum nucleons. The results suggest that the effect of Fermi motion is extremely important if one is to draw conclusions regarding the role of SRCs in the valence EMC effect.

Schmookler et al. [26] assumed that the structure function of nucleus $A$ could be decomposed into contributions from unmodified mean-field protons and neutrons and contributions from $n p$ pairs in SRCs with modified structure functions:

$$
\begin{aligned}
F_{2}^{A} & =\left(Z-n_{\mathrm{SRC}}^{A}\right) F_{2}^{p}+\left(N-n_{\mathrm{SRC}}^{A}\right) F_{2}^{n}+n_{\mathrm{SRC}}^{A}\left(F_{2}^{p *}+F_{2}^{n *}\right) \\
& =Z F_{2}^{p}+N F_{2}^{n}+n_{\mathrm{SRC}}^{A}\left(\Delta F_{2}^{p}+\Delta F_{2}^{n}\right),
\end{aligned}
$$

where $n_{\mathrm{SRC}}^{A}$ is the number of $n p$ pairs in the nucleus $A$ and the structure functions depend on the Bjorken $x$ variable and the exchanged four-momentum squared $Q^{2}$. The off-shell nucleon structure modifications are denoted

$$
\Delta F_{2}^{N}=F_{2}^{N *}-F_{2}^{N}, \quad N=p, n,
$$

where $F_{2}^{N *}$ is the averaged modified structure function for nucleons in SRC pairs. Inherent in this formulation is the assumption that all of the modifications of the nucleon structure can be absorbed in the form of the 
off-shell corrections $\Delta F_{2}^{N}$. The relative modification of the structure functions of nucleons in $n p$ SRC pairs,

$$
\frac{n_{\mathrm{SRC}}^{d}\left(\Delta F_{2}^{p}+\Delta F_{2}^{n}\right)}{F_{2}^{d}}=\frac{F_{2}^{A} / F_{2}^{d}-(Z-N) F_{2}^{p} / F_{2}^{d}-N}{(A / 2) a_{2}-N},
$$

where $a_{2}=(2 / A)\left(n_{\mathrm{SRC}}^{A} / n_{\mathrm{SRC}}^{d}\right)$, was extracted from the EMC data as a universal function for all nuclei. Applying Eq. (1) to a deuteron target, this universal function is related to the deuteron structure function by

$$
R \equiv \frac{F_{2}^{d}-\left(F_{2}^{p}+F_{2}^{n}\right)}{F_{2}^{d}}=\frac{n_{\mathrm{SRC}}^{d}\left(\Delta F_{2}^{p}+\Delta F_{2}^{n}\right)}{F_{2}^{d}} .
$$

For comparison with the phenomenological analysis of Ref. [26], we consider the nuclear structure function within the relativistic impulse approximation, in which the nuclear DIS takes place via incoherent scattering from bound, offshell nucleons [27-37]. One can show that in this case the nuclear $F_{2}^{A}$ structure function can be written as a sum of a convolution term involving on-shell nucleon structure functions and an off-shell correction [28]:

$F_{2}^{d}(x)=\left(f_{p / d} \otimes F_{2}^{p}+f_{n / d} \otimes F_{2}^{n}\right)(x)+F_{2}^{\mathrm{d}(\mathrm{off})}(x)$,

where the convolution symbol $\otimes$ is defined by the integral $\left(f \otimes F_{2}\right)(x)=\int_{x}^{A} d y f(y) F_{2}(x / y)$ and, for simplicity, we have suppressed the $Q^{2}$ dependence in the functions. The smearing function $f_{p / d}=f_{n / d} \equiv f_{N / d}$ describes the distribution of nucleons in the deuteron carrying a fraction $y=\left(p_{0}+p_{z}\right) / M$ of the deuteron's light-cone momentum:

$$
f_{N / d}(y)=\int_{-\infty}^{p_{\max }^{2}} d p^{2} \widetilde{f}_{N / d}\left(y, p^{2}\right),
$$

where $p^{2}=p_{0}^{2}-\boldsymbol{p}^{2}$ is the invariant mass squared of the struck nucleon, with the maximum value given by $p_{\max }^{2}=\left[y(1-y / 2) M_{d}^{2}-y M^{2}\right] /(2-y)$. Since the spectator nucleon is on shell with energy $E_{p}=\sqrt{M^{2}+p^{2}}$, conservation of four-momentum requires the struck nucleon to have energy $p_{0}=M_{d}-E_{p}$ and go far off shell when the three-momentum associated with SRCs is high. The nucleon momentum distribution can be straightforwardly computed from the relativistic (four-component) deuteron wave function [38]:

$$
\widetilde{f}_{N / d}\left(y, p^{2}\right) \propto\left|\psi_{d}(p)\right|^{2},
$$

and formalism reproduces the nonrelativistic expressions found in earlier work $[7,27,30,39]$. Note that the deuteron momentum fraction carried by the struck nucleon, $y$, carries the information that the struck nucleon is far off-mass-shell when it is involved in a SRC.
On the other hand, the off-shell term identified in Ref. [28], $F_{2}^{d(\text { off })}$, isolates the dependence on the medium modification of the nucleon structure and is more model dependent. One should caution that the off-shell term $F_{2}^{d(\text { off })}$ in Eq. (5) [as well as the off-shell term $\Delta F_{2}^{N}$ in Eq. (2)] is not physical, as one can, in principle, move strength between the different terms in Eq. (5). Nevertheless, in the literature, a number of attempts have been made to estimate the nucleon off-shell modifications consistently within specific models.

Working within a covariant quark-hadron framework, Melnitchouk et al. [28,29] computed $F_{2}^{d}$ using relativistic nucleon-deuteron vertex functions [38] (see also [40,41]). These include $P$-wave admixtures and take into account Lorentz scalar and vector interactions in the deuteron. For the nucleon itself, this approach used a spectator quark model parametrizing off-shell nucleon-quark-spectator vertex functions for spin-0 and spin-1 diquark spectators. The off-shell correction $F_{2}^{d(\text { off })}$ was given by a sum of nonfactorized terms depending on the $P$-state wave functions and the nucleon virtuality $v\left(p^{2}\right) \equiv\left(p^{2}-M^{2}\right) / M^{2}$, where $M$ is the nucleon mass. Integrating over all $p^{2}$, the off-shell effects in this model were found to be $\lesssim 1 \%-2 \%$ in the relevant range of $x$ [29].

A similar formulation by Kulagin et al. $[30,35,42,43]$ expands the off-shell nucleon scattering amplitude in powers of the nucleon momentum $\boldsymbol{p}$, allowing the nuclear structure function to be expressed as a generalized convolution of the nuclear spectral function and a $p^{2}$ dependent nucleon structure function $\widetilde{F}_{2}^{N}$ :

$$
F_{2}^{d}(x)=\sum_{N} \int d y d p^{2} \widetilde{f}_{N / d}\left(y, p^{2}\right) \widetilde{F}_{2}^{N}\left(x / y, p^{2}\right) .
$$

Expanding the off-shell nucleon function in a Taylor series around $v=0$, one finds

$$
\widetilde{F}_{2}^{N}\left(x, p^{2}\right)=F_{2}^{N}(x)\left[1+v\left(p^{2}\right) \delta f(x)+\mathcal{O}\left(v^{2}\right)\right] .
$$

The off-shell correction term $F_{2}^{d(\text { off })}$ in Eq. (5) can then be written as a convolution of an off-shell smearing function $f_{N / d}^{\text {(off) }}$ and the nucleon off-shell function $\delta f[37,44]$ :

$$
F_{2}^{d(\text { off })}(x)=\sum_{N}\left(f_{N / d}^{(\text {off })} \otimes\left[F_{2}^{N} \cdot \delta f\right]\right)(x),
$$

where

$$
f_{N / d}^{(\text {off })}(y)=\int d p^{2} v\left(p^{2}\right) \widetilde{f}_{N / d}\left(y, p^{2}\right) .
$$

Assuming that the off-shell structure function $\widetilde{F}_{2}^{N}$ has a spectral representation, the off-shell dependence of the quark spectral function was modeled in terms of the $p^{2}$ dependence of the ultraviolet cutoff parameter regularizing 


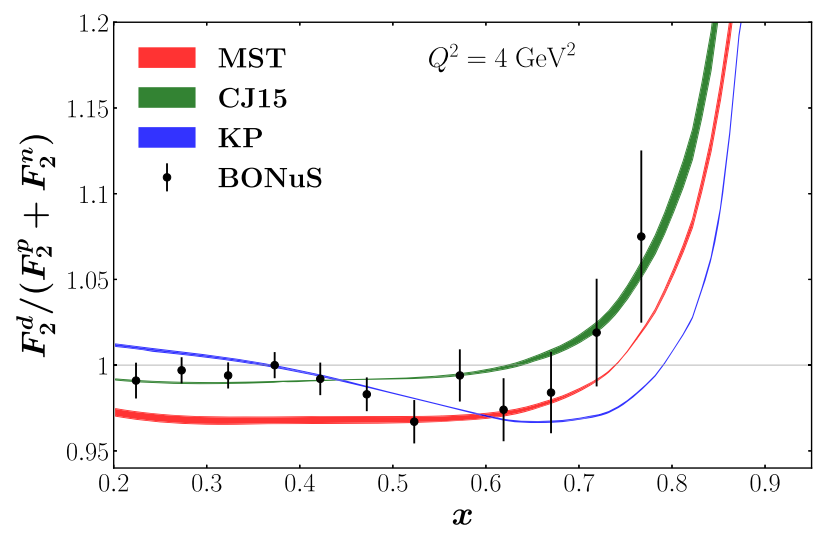

FIG. 1. Ratio of deuteron to isoscalar nucleon structure functions $F_{2}^{d} /\left(F_{2}^{p}+F_{2}^{n}\right)$ from the relativistic spectator model (MST) [29] (red band), the CJ15 global QCD analysis [47] (green band), and the Kulagin-Petti (KP) analysis [35] (blue band), compared with data from the Barely Offshell Nucleon Structure experiment [50] (black circles). The bands represent results using the Paris [51] and WJC-2 [52] deuteron wave functions.

the integration over the quark momentum [35,45], which it was argued could be related to the confinement radius and the amount of nucleon swelling in the nuclear medium [46]. Alternatively, rather than rely on models to estimate the offshell function $\delta f$, several analyses have more recently sought to parametrize the function phenomenologically and determine the parameters directly from a global QCD analysis of high-energy proton and deuteron data [36,47].

The results of these analyses for the nuclear EMC ratio in the deuteron,

$$
R_{\mathrm{EMC}}^{d} \equiv \frac{F_{2}^{d}}{F_{2}^{p}+F_{2}^{n}}=\frac{1}{1-R} \approx 1+R \quad \text { for } R \ll 1,
$$

are illustrated in Fig. 1 at a fixed $Q^{2}=4 \mathrm{GeV}^{2}$ and compared with data from the Barely Offshell Nucleon Structure experiment at Jefferson Lab [48-50]. This experiment measured tagged low-momentum protons in coincidence with scattered electrons in DIS from the deuteron, with $Q^{2}$ ranging from $1.2 \mathrm{GeV}^{2}$ at the lowest $x$ value shown up to $4.0 \mathrm{GeV}^{2}$ at the highest $x$. Within the uncertainties quoted by the experiment (in Fig. 1, the statistical and systematic uncertainties have been added in quadrature), the inclusive data cannot at present definitively discriminate between the different off-shell behaviors in the models.

The essential difference for our purposes between all the models [26,28,29,35,46,47,53] discussed above is the relative amount of the valence EMC effect that is attributed to nucleons with exceptionally high momentum, especially those in SRCs. Whereas in the convolution model of Eq. (5) the off-shell effects are just one source of difference between the free-nucleon and nuclear structure functions (with kinematic and binding corrections being vital), the

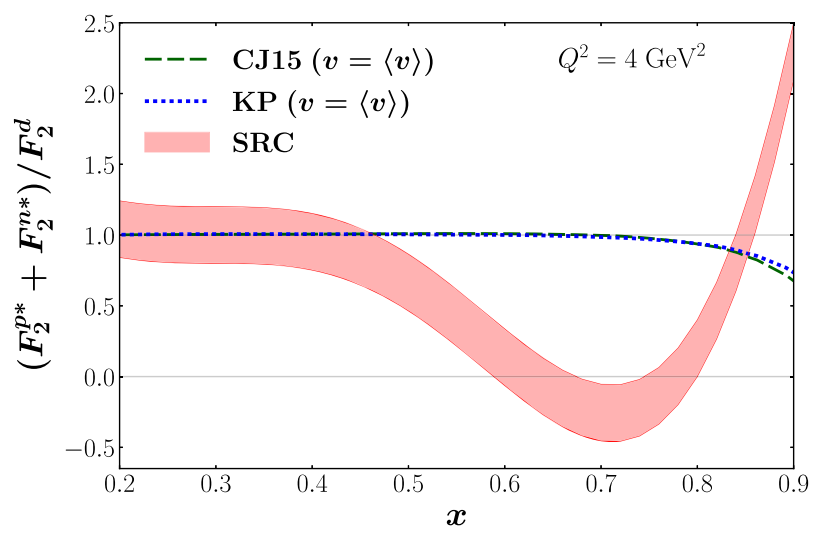

FIG. 2. Ratio of off-shell nucleon structure function to the deuteron structure function $\left(F_{2}^{p *}+F_{2}^{n *}\right) / F_{2}^{d}$ for the models discussed in Fig. 1. The ratio for the SRC model is computed from Eq. (13) (the red band represents the uncertainty on the SRC parametrization from Ref. [26]), while for the CJ15 and KP models the off-shell nucleon functions are computed from Eq. (9) at an average nucleon virtuality $v=\langle v\rangle$.

model in Eq. (1) represents the most extreme scenario, whereby all of the nuclear corrections are attributed to the "universal modification function" associated with nucleons in SRCs [Eq. (2)].

This is more dramatically illustrated in Fig. 2, where we show the ratio of the (unphysical) off-shell nucleon structure function to the deuteron structure function, $\left(F_{2}^{p *}+F_{2}^{n *}\right) / F_{2}^{d}$. For the SRC model, from Eqs. (2) and (4), this can be computed from the ratio $R$ and the number $n_{\mathrm{SRC}}^{d}$ of $n p$ pairs in the deuteron:

$$
\frac{F_{2}^{p *}+F_{2}^{n *}}{F_{2}^{d}}=1+\frac{R}{n_{\mathrm{SRC}}^{d}}\left(1-n_{\mathrm{SRC}}^{d}\right) .
$$

The probability of finding nucleons in an SRC pair in the deuteron can be evaluated by integrating the deuteron wave function over large relative momenta:

$$
n_{\mathrm{SRC}}^{d}=\int_{p_{F}}^{\infty} d|\boldsymbol{p}| \boldsymbol{p}^{2}\left|\psi_{d}(p)\right|^{2} .
$$

Taking a typical choice for the (assumed) universal point of demarcation between low-momentum nucleons and those in SRCs, denoted $p_{F}$, to be $p_{F}=300 \mathrm{MeV}$, one finds $n_{\mathrm{SRC}}^{d}=3.4 \%$ for the Paris deuteron wave function [51], while for the relativistic WJC-2 deuteron wave function [52] one has $n_{\text {SRC }}^{d}=3.8 \%$ (see also Ref. [53]). The resulting ratio is close to unity at low $x$ but then decreases dramatically to even go negative at $x \sim 0.6-0.8$, before increasing again at higher $x$ values to mimic the effect of Fermi motion.

In contrast, the off-shell nucleon structure functions in the convolution approach appear under the integral in Eq. (8). Defining the average value of the virtuality $v$ by 


$$
\langle v\rangle=\int d y f_{N / d}^{(\mathrm{off})}(y)
$$

we find $\langle v\rangle=-4.3 \%$ for the Paris wave function and $-5.0 \%$ for the WJC-2 wave function. The ratio in Fig. 2 can then be evaluated according to

$\frac{F_{2}^{p *}+F_{2}^{n *}}{F_{2}^{d}}=\frac{\left\langle\widetilde{F}_{2}^{p}+\widetilde{F}_{2}^{n}\right\rangle}{F_{2}^{d}}=\frac{\left(F_{2}^{p}+F_{2}^{n}\right)(1+\delta f\langle v\rangle)}{F_{2}^{d}}$.

Since the off-shell function $\delta f$ is generally $\lesssim \mathcal{O}(1)$, the ratio (16) deviates from unity only very slightly due to the small value of $\langle v\rangle$. In fact, the ratios in Fig. 2 for the convolution models are basically the inverse of the EMC effect ratios shown in Fig. 1, with deviations from this proportional to $\delta f\langle v\rangle$ that are negligible.

While not ruled out phenomenologically by the inclusive structure function measurements, the interpretation of a negative off-shell nucleon structure function in the SRC model, which appears already for $x \gtrsim 0.6$, is problematic. The striking contrast between the two pictures illustrated in Fig. 2 suggests the need to further explore whether the offshell scenarios can be distinguished in specific regions of $p^{2}$, such as those typically associated with SRCs.

In fact, the formulation of the convolution models in Eqs. (5) and (8) explicitly in terms of the momentumdependent functions makes it possible to separate the contributions to the deuteron structure function from the low- and high-momentum regions:

$$
F_{2}^{d}=\left.F_{2}^{d}\right|_{p<p_{F}}+\left.F_{2}^{d}\right|_{p>p_{F}}
$$

where the first and second terms represent contributions to the integrals in Eqs. (6), (8), and (11) from below and above the boundary momentum $p_{F}$, respectively. If SRCs are indeed responsible for the observed valence EMC effect, as hypothesized in Ref. [26], one should find that the $p>p_{F}$ term in Eq. (17) plays a leading role in generating the differences between the nuclear and free-nucleon structure functions in the valence region.
To test this hypothesis, we use Eq. (17) to decompose the ratio $R$ in Eq. (4) into low-momentum and high-momentum components:

$$
R=R_{p>p_{F}}+R_{p<p_{F}},
$$

where

$$
\begin{aligned}
R_{p>p_{F}} & =\frac{\left.F_{2}^{d}\right|_{p>p_{F}}-n_{\mathrm{SRC}}^{d}\left(F_{2}^{p}+F_{2}^{n}\right)}{F_{2}^{d}}, \\
R_{p<p_{F}} & =\frac{\left.F_{2}^{d}\right|_{p<p_{F}}-\left(1-n_{\mathrm{SRC}}^{d}\right)\left(F_{2}^{p}+F_{2}^{n}\right)}{F_{2}^{d}} .
\end{aligned}
$$

The individual contributions $R_{p>p_{F}}$ and $R_{p<p_{F}}$, as well as the total ratio $R$, are shown in Fig. 3 for each of the three microscopic models considered [29,35,47]. As may be expected from Eq. (12), for each model the shape of the total $R$ ratio follows that of the $R_{\mathrm{EMC}}^{d}$ ratio in Fig. 1. On the other hand, the relative contributions to the ratio from the low- and high-momentum regions reveal features that are not evident in the integrated quantities.

In particular, for the MST model [29] in Fig. 3(a), one finds that in the valence EMC effect region $0.3 \lesssim x \lesssim 0.7$ less than half of the strength comes from the highmomentum $p>p_{F}$ region at the lower $x$ values and even less at the higher- $x$ end of the range. For even larger $x$ values, $x \rightarrow 1$, the rise in $R$ due to Fermi motion is also more strongly associated with the $p<p_{F}$ region.

For the CTEQ-Jefferson Lab (CJ15) case in Fig. 3(b), the overall nuclear EMC effect is found to be the smallest, as also evident from Fig. 1 , with $\approx 1 / 3$ of the magnitude of that in the MST model. However, within the small absolute size of the total effect, essentially $100 \%$ comes from the $p<p_{F}$ region for $x \lesssim 0.5$, while at larger $x$ values, $x \sim 0.6-0.7$, the low- and high- $p$ contributions largely cancel. In contrast, for the KP model in Fig. 3(c), where the EMC effect follows the general characteristics of the effect in heavy nuclei, the deviation from zero is greatest for $x \approx 0.6-0.7$, where it is completely dominated by the lowmomentum, $p<p_{F}$, contributions.

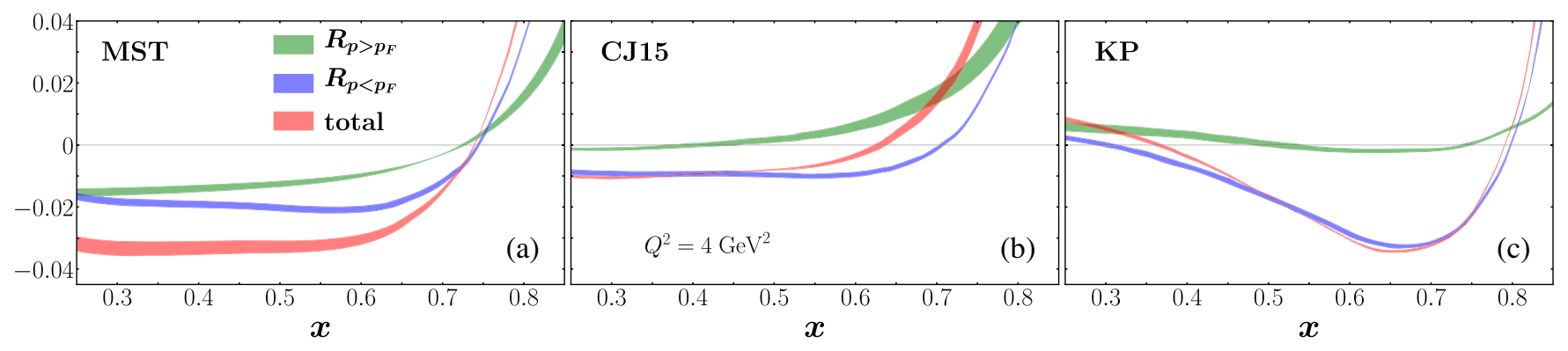

FIG. 3. Contributions to the ratio $R=\left[F_{2}^{d}-\left(F_{2}^{p}+F_{2}^{n}\right)\right] / F_{2}^{d}$ from the regions $p>p_{F}$ (green bands), $p<p_{F}$ (blue bands), and the total (red bands), for the (a) MST [29], (b) CJ15 [47], and (c) KP [35] models for the nucleon off-shell corrections at $Q^{2}=4 \mathrm{GeV}^{2}$. The bands envelop the results obtained using the nonrelativistic Paris [51] and the relativistic WJC-2 [52] deuteron wave functions. 
The inescapable conclusion to be drawn from the results in Fig. 3 is that, even though the shapes and magnitudes of the EMC effect differ considerably between the different models, in no case do the SRCs give the dominant, let alone the total, EMC effect. The total valence EMC effect either is dominated by the low-momentum $p<p_{F}$ components (as in the KP model and in the CJ15 model at lower $x$ ) or received approximately similar contributions from both regions (as in the MST model and in the CJ15 case at higher $x$ ). This finding is independent of the choice of deuteron wave function, with essentially the same results obtained for the widely used Paris wave function [51] and the more recent relativistic WJC-2 wave functions [52].

We have also investigated the effect of the variation of the $\boldsymbol{p}$ boundary, in particular, when this boundary momentum is raised to $p_{F}=400 \mathrm{MeV}$. In this case, the number of nucleons in SRCs decreases slightly to $n_{\mathrm{SRC}}^{d}=2.0 \%$ for the Paris wave function and $2.6 \%$ for the WJC-2 wave function. Compared with results obtained with $p_{F}=300 \mathrm{MeV}$ as the boundary for SRCs, the magnitude of the $p<p_{F}$ contribution increases for the larger $p_{F}$ and becomes closer to the total result in each case in Fig. 3. This can be expected from the definition of $R_{p>p_{F}}$ and $R_{p<p_{F}}$ in Eqs. (19): When $p_{F} \rightarrow 0, R_{p>p_{F}} \rightarrow R$ and $R_{p<p_{F}} \rightarrow 0$, while when $p_{F} \rightarrow \infty, R_{p>p_{F}} \rightarrow 0$ and $R_{p<p_{F}} \rightarrow R$.

Clearly, this analysis does not support the hypothesis that there is a causal connection between nucleons residing in SRCs and the EMC effect. Instead, it suggests that the effect in the deuteron is dominated by binding and, to a lesser extent, off-shell effects associated with nucleons in the region $p<p_{F}$. It is crucial to this conclusion that neglecting the effect of Fermi motion is a particularly poor approximation, especially for nucleons in SRCs, and in no model do SRCs give more than half the EMC effect in deuterium.

Future avenues to explore the origin of the nuclear EMC effect include semi-inclusive DIS on the deuteron and other light nuclei, with tagging of recoil protons and neutrons to leverage the virtuality of the scattered nucleon [54]. Asymmetric nuclei can provide additional information on the isospin dependence of the off-shell effects, which has been raised in recent studies of DIS from ${ }^{3} \mathrm{He}$ [37]. Finally, there are dramatically different predictions for the spin $[21,24]$ and flavor [23] dependence of the EMC effect in microscopic models and those predicted from the SRC picture. Data from upcoming experiments at Jefferson Lab or the future Electron-Ion Collider could be instrumental in finally resolving the long sought-after origin of the nuclear EMC effect.

This work was supported by the Australian Research Council through the Discovery Projects No. DP151103101 and No. DP180100497 (A. W. T.) and the U.S. Department of Energy Contract No. DE-AC05-06OR23177, under which Jefferson Science Associates, LLC operates Jefferson Lab (W. M.).
[1] J. J. Aubert et al., Phys. Lett. 123B, 275 (1983).

[2] J. Ashman et al., Phys. Lett. B 202, 603 (1988).

[3] A. C. Benvenuti et al., Phys. Lett. B 189, 483 (1987).

[4] J. Gomez et al., Phys. Rev. D 49, 4348 (1994).

[5] D. F. Geesaman, K. Saito, and A. W. Thomas, Annu. Rev. Nucl. Part. Sci. 45, 337 (1995).

[6] P. R. Norton, Rep. Prog. Phys. 66, 1253 (2003).

[7] R. P. Bickerstaff and A. W. Thomas, J. Phys. G 15, 1523 (1989).

[8] E. L. Berger and F. Coester, Annu. Rev. Nucl. Part. Sci. 37, 463 (1987).

[9] N. Fomin et al., Phys. Rev. Lett. 108, 092502 (2012).

[10] J. Seely et al., Phys. Rev. Lett. 103, 202301 (2009).

[11] F. E. Close, R. G. Roberts, and G. G. Ross, Phys. Lett. 129B, 346 (1983).

[12] F. E. Close, R. G. Roberts, and G. G. Ross, Phys. Lett. 142B, 202 (1984).

[13] C. H. Llewellyn Smith, Phys. Lett. 128B, 107 (1983).

[14] M. Ericson and A. W. Thomas, Phys. Lett. 128B, 112 (1983).

[15] E. L. Berger, F. Coester, and R. B. Wiringa, Phys. Rev. D 29, 398 (1984).

[16] G. V. Dunne and A. W. Thomas, Nucl. Phys. A455, 701 (1986).

[17] L. L. Frankfurt and M. I. Strikman, Phys. Rep. 160, 235 (1988).

[18] A. W. Thomas, A. Michels, A. W. Schreiber, and P. A. M. Guichon, Phys. Lett. B 233, 43 (1989).

[19] K. Saito, A. Michels, and A. W. Thomas, Phys. Rev. C 46, R2149 (1992).

[20] H. Mineo, W. Bentz, N. Ishii, A. W. Thomas, and K. Yazaki, Nucl. Phys. A735, 482 (2004).

[21] I. C. Cloët, W. Bentz, and A. W. Thomas, Phys. Lett. B 642 , 210 (2006).

[22] J. R. Smith and G. A. Miller, Phys. Rev. C 65, 055206 (2002).

[23] I. C. Cloët, W. Bentz, and A. W. Thomas, Phys. Rev. Lett. 109, 182301 (2012).

[24] A. W. Thomas, Int. J. Mod. Phys. E 27, 1840001 (2018).

[25] M. Duer et al., Nature (London) 560, 617 (2018).

[26] B. Schmookler et al., Nature (London) 566, 354 (2019).

[27] F. Gross and S. Liuti, Phys. Rev. C 45, 1374 (1992).

[28] W. Melnitchouk, A. W. Schreiber, and A. W. Thomas, Phys. Rev. D 49, 1183 (1994).

[29] W. Melnitchouk, A. W. Schreiber, and A. W. Thomas, Phys. Lett. B 335, 11 (1994).

[30] S. A. Kulagin, G. Piller, and W. Weise, Phys. Rev. C 50, 1154 (1994).

[31] I. R. Afnan, F. R. P. Bissey, J. Gomez, A. T. Katramatou, S. Liuti, W. Melnitchouk, G. G. Petratos, and A. W. Thomas, Phys. Rev. C 68, 035201 (2003).

[32] E. Pace, G. Salme, S. Scopetta, and A. Kievsky, Phys. Rev. C 64, 055203 (2001).

[33] M. M. Sargsian, S. Simula, and M. I. Strikman, Phys. Rev. C 66, 024001 (2002).

[34] A. Rinat and M. Taragin, Phys. Lett. B 551, 284 (2003).

[35] S. A. Kulagin and R. Petti, Nucl. Phys. A765, 126 (2006).

[36] S. A. Kulagin and R. Petti, Phys. Rev. C 82, 054614 (2010).

[37] A. J. Tropiano, J. J. Ethier, W. Melnitchouk, and N. Sato, Phys. Rev. C 99, 035201 (2019). 
[38] W. W. Buck and F. Gross, Phys. Rev. D 20, 2361 (1979).

[39] C. Ciofi degli Atti and S. Liuti, Phys. Rev. C 41, 1100 (1990).

[40] F. Gross and A. Stadler, Phys. Lett. B 657, 176 (2007).

[41] F. Gross and A. Stadler, Phys. Rev. C 82, 034004 (2010).

[42] S. A. Kulagin, W. Melnitchouk, G. Piller, and W. Weise, Phys. Rev. C 52, 932 (1995).

[43] S. A. Kulagin and W. Melnitchouk, Phys. Rev. C 77, 015210 (2008).

[44] P. J. Ehlers, A. Accardi, L. T. Brady, and W. Melnitchouk, Phys. Rev. D 90, 014010 (2014).

[45] J. J. Ethier and W. Melnitchouk, Phys. Rev. C 88, 054001 (2013).

[46] A. Accardi, W. Melnitchouk, J. F. Owens, M. E. Christy, C. E. Keppel, L. Zhu, and J. G. Morfin, Phys. Rev. D 84, 014008 (2011).
[47] A. Accardi, L. T. Brady, W. Melnitchouk, J. F. Owens, and N. Sato, Phys. Rev. D 93, 114017 (2016).

[48] N. Baillie et al., Phys. Rev. Lett. 108, 142001 (2012); 108, 199902(E) (2012).

[49] S. Tkachenko et al., Phys. Rev. C 89, 045206 (2014); 90, A059901 (2014).

[50] K. A. Griffioen et al., Phys. Rev. C 92, 015211 (2015).

[51] M. Lacombe, B. Loiseau, R. Vinh Mau, J. Cote, P. Pires, and R. de Tourreil, Phys. Lett. 101B, 139 (1981).

[52] F. Gross and A. Stadler, Phys. Rev. C 78, 014005 (2008); 82, 034004 (2010).

[53] E. P. Segarra, A. Schmidt, T. Kutz, D. W. Higinbotham, E. Piasetzky, M. Strikman, L. B. Weinstein, and O. Hen, Phys. Rev. Lett. 124, 092002 (2020).

[54] W. Melnitchouk, M. Sargsian, and M. Strikman, Z. Phys. A 359, 99 (1997). 